\title{
ASPECTOS SUSTANCIALES DEL NE BIS IN ÍDEM DEL ESTATUTO DE ROMA (ER) DE 1998 EN EL DERECHO INTERNO.
}

Jaime Alberto Sandoval Mesa*

Fecha de Recibido: 15 de octubre de 2010

Fecha de Aprobación: 15 de marzo de 2011

Articulo de Resultado de Investigación

\begin{abstract}
Resumen
El presente artículo es un producto de investigación elaborado en el proyecto incidencia de los instrumentos internacionales en materia penal que se adelanta en la Universidad Militar Nueva Granada. El documento analiza las dudas que se originan a raíz de la influencia del Estatuto de Roma de 1998, frente al principio de legalidad en su manifestación de cosa juzgada (non bis in ídem) y en la praxis que parece asumirse en la aplicación de dicho instrumento, en los casos concretos que pueden ser objeto de competencia e investigación, un poco distinta de nuestra tradición continental. En esta última parte, es importante tener en cuenta tales consecuencias, toda vez que frente al eventual ejercicio de la competencia e investigación, es cuando en realidad, se puede determinar si en virtud de la ocurrencia de una conducta, corresponde en su valoración en la CPI, a los efectos que se producen en el derecho interno.
\end{abstract}

\section{Palabras claves}

Cosa Juzgada material, ne bis in ídem, legalidad, concurso de conductas punibles, competencia, investigación, Admisibilidad.

\section{BASIC ASPECTS OF THE NE BIS IN DEM OF THE ROME STATUTE (ER) OF 1998 ON THE INTERIOR LAW}

\begin{abstract}
This article is a research product developed in the project impact of international instruments in criminal matters which is ahead in Nueva Granada military University. The paper analyses the doubts that arise as a result of the influence of the Rome Statute of 1998, compared with the principle of legality in their demonstration of ne bis in idem and in the practice that seems to assume in the implementation of that instrument, in the specific cases that may be subject to competition and research, a little different from our continental tradition. In this last part, it is important to take into account such implications, given that with the eventual exercise of competition and research, it is when
\end{abstract}

Docente e Investigador Facultad de Derecho Universidad Militar Nueva Granada Bogotá. Magíster en Derecho Penal. Universidad Santo Tomás Bogotá D.C., - Universidad de Salamanca, España. Especialista en Derecho Público de la Universidad Nacional de Colombia. Estudios de Posgrado en Derecho Constitucional Universidad de Salamanca, España. Abogado y Especialista en Derecho Penal Universidad Santo Tomás Bogotá D.C. jaime.sandoval@unimilitar.edu.co; jaisandoval@yahoo.com. 
in reality, can be if by virtue of the occurrence of conduct, it is in its valuation at the ICC, to the effects in the internal law.

\title{
Key words
}

$\mathrm{Ne}$ bis in idem, legality, punishable conduct, competition, research, admissibility contest

\section{ASPECTOS SUBSTANCIAIS DO NE BIS IN ÍDEM DO ESTATUTO DE ROMA (ER) DE 1998 NO DIREITO INTERNO}

\begin{abstract}
Resumo
O presente artigo é resultado de pesquisa elaborada dentro do projeto Incidência dos Instrumentos Internacionais em Matéria Penal, que vem sendo realizado na Universidade Militar Nova Granada. O documento analisa as dúvidas que surgem como conseqüência da influência do Estatuto de Roma de 1998 no principio de legalidade em sua manifestação de coisa julgada (non bis in idem), e na práxis que parece ser assumida na aplicação de tal instrumento em casos concretos que podem ser objeto de competência e investigação, um pouco diferente da nossa tradição continental. Nesta ultima parte, é importante considerar tais conseqüências pois, na realidade, sempre é diante do eventual exercício da competência e investigação que pode ser determinado se diante da ocorrência de uma conduta corresponde sua avaliação na CPI para os efeitos gerados no Direito interno
\end{abstract}

\section{Palavras-chave}

Coisa Julgada material, ne bis in idem, legalidade, exame de condutas puníveis, competência, pesquisa, admissibilidade.

\section{ASPECTOS SUSTANCIALES DEL NON BIS IN ÍDEM DEL ESTATUTO DE ROMA (ER) DE 1998 EN EL DERECHO INTERNO.}

En primer lugar, resulta pertinente analizar los elementos que se configuran en el concepto de ne bis in ídem(cosa juzgada), que pertenecen ala orientación continental y en forma indefectible, al ámbito de la valoración de la conducta frente a los supuestos establecidos en el artículo $5^{\circ} \mathrm{del}$ Estatuto de Roma (en adelante ER.). Así mismo, resulta conveniente determinar los elementos que lo configuran para verificar, finalmente, si la misma corresponde a uno o a varios de los crímenes comprendidos en la norma citada. Pues bien este objeto de valoración frente al derecho interno, será desarrollado en el presente documento, mediante el método deductivo, hermenéutico y analítico, con base en herramientas comparativas, propositivas y descriptivas, conforme a la siguiente exposición.

\subsection{Situaciones particulares del nullum crimen sine lege, en relación con el non bis in idem sustancial - Determi- nación de la Conducta y de la Pena.}

En primer lugar, es preciso mencionar que del estatuto de Roma de 1998, se derivan dificultades de interpretación de la lex certa y stricta en virtud de algunas diferencias que se presentan con respecto al tradicional principio de legalidad de tipo continental europeo y a su turno, generan efectos prominentes con respecto al non bis in ídem (Art. 20 ER.). Sobre este aspecto, se puede prever que de la determinación que se realiza respecto del nullum crimen sine lege del Estatuto de Roma (Art. 22 E.R.), se pueden generar circunstancias de gran amplitud en el derecho interno, debido a algunos factores hermenéuticos de difícil concreción, tales como los descritos en el numeral $3^{\circ}$ de la norma comentada, que señala 
la interpretación de crímenes internacionales independientemente del Estatuto de Roma (ER. 22.3) conforme al derecho internacional.

Sobre el particular, González Cussac y Gorriz Royo, (GONZALEZ \& GORRIZ: 2003: 234) advierten que la amplitud de determinación del art. 22 ER., implica múltiples consecuencias frente a las conductas descritas en el Art. $5^{\circ}$ ER., las cuales causan innumerables efectos con respecto al non bis in ídem y a su interpretación (art. 20 E.R.). Así mismo señalan, que el fenómeno anterior, genera variadas relaciones que pueden plantearse a raíz del $\mathrm{ER}$, no sólo, debido a la proclamación del principio del ne bis in ídem que no desarrolla aspectos sustanciales, sino que su orientación procesal, no proporciona los concretos criterios materiales de solución en casos de concursos de conductas punibles, solapamientos normativos etc., de acuerdo con lo estatuido en Art. $5^{\circ} \mathrm{ER}$. (GONZALEZ \& GORRIZ: 2003: 235).

En efecto, el non bis in ídem sustancial, en el ER, desde un ámbito sistémico, carece de elementos en cuanto a la determinación de penas y situaciones de concurrencia delictiva, (GONZALEZ \& GORRIZ: 2003: 235) pues en el art. 78 E.R. se apela para su imposición, únicamente a la gravedad del crimen y a las circunstancias del condenado, aspecto que no precisa la solución de los casos de concursos de infracciones. En esencia, éste artículo menciona que se impondrá una pena por cada uno de los delitos cometidos y además se recoge una "regla concursal" atendiendo la previsión de que se imponga una pena común, sin que sean especificados los criterios para atender esta exigencia. Tal aspecto se ve reflejado tanto en los supuestos del art. (77.1.a) - pena privativa que no exceda de 30 años- y (77.1. b) - pena de prisión a perpetuidad E.R. ${ }^{1}$

1 En el Estatuto de Roma el recurso al derecho nacional para la determinación de la pena no resulta vinculante para los fines previstos en la determinación de la sanción. Así mismo, los criterios de la gravedad del hecho y las circunstancias atenuantes y agravantes, pueden ser tenidas en cuenta en el momento de fijar una
Sobre esto hay que dejar en claro que pese a que los temas relativos a las formas concursales de la pena, guardan relación con el ámbito de discusión tratado en este escrito, de todas formas hacen parte del principio, nulla poena sine lege (Art. 23 ER.) que no será objeto de tratamiento in extenso en el presente debate. Sin embargo, es de destacar desde este primer acercamiento, la falta de concreción para determinar una pena específica bajo unos criterios explícitos de punibilidad, lo cual permite identificar otra de las consecuencias de la determinación de la conducta y por ende del non bis in ídem material abordado ${ }^{2}$.

En todo caso, desde este punto de vista, es de observar que el sistema interno, merece un análisis particular, en la medida en que las consecuencias del principio incorporado en nuestra legislación penal, obedece a todo lo contrario a lo establecido en el Estatuto de Roma. En efecto, el principio consagrado sobre la imposición de penas y la naturaleza de las mismas, tanto en los arts. 28 y 29, de la Constitución, así como también en los artículos $6^{\circ}, 34$ y ss., del C. P., se sujetan a figuras expresas sobre los criterios tendientes a determinar el alcance y límites precisos para su fijación. Desde aquí aparece una primera inconsistencia entre el Derecho propio de la CPI y el derecho interno, precisamente desde el punto de vista material de la consideración y fijación de la sanción en la relación delito-pena.

Lo anterior parece tener soporte, en la interpretación que debe hacerse del art. 21 E.R. en tales circunstancias, toda vez que, sólo puede acudirse a los principios de derecho interno, una vez agotados los principios establecidos en el Estatuto, en las reglas de procedimiento y prueba y en

única condena, solución que se deriva no solamente del reglamento de procedimiento y prueba, sino también de la jurisprudencia del Tribunal de la Ex Yugoeslavia entre otros. FRONZA Emanuela. Capitulo V, Las Sanciones. Introducción al Derecho Penal Internacional. Traductor Yezid Viveros Castellanos. Edit. Kimpres-Universidad Libre, Bogotá 2009 pp. 334 a 337.

2 Sobre este aspecto, tampoco aparece ninguna referencia al Derecho Penal Nacional. FRONZA Emanuela. P. 346. 
los principios sobre derechos humanos que rigen la materia. Con lo anterior, se comienza a vislumbar el carácter de fuente terciaria que ostenta el derecho interno y su reducida aplicación frente a la primacía del instrumento analizado. (COSTTI \& FRONZA: 2009: 101-112)

El presupuesto anterior, se observa aun con mayor trascendencia, si se tiene en cuenta la interpretación de la Corte Constitucional, frente a los efectos de la fijación de la sanción por parte de la CPI frente a los límites internos. En estos casos indicó lo siguiente: “(...) En el artículo 77.1, literal b) el Estatuto faculta a la Corte Penal Internacional a imponer la pena de reclusión a perpetuidad. Este tratamiento diferente de la prohibición de la prisión perpetua que consagra el artículo 34 de la Carta, fue autorizado el Acto Legislativo No. 02 de 2001, pero no habilita a las autoridades nacionales a aplicar este tipo de pena cuando juzguen alguno de los crímenes señalados en el Estatuto de Roma". (CORTE CONSTITITUCIONAL COLOMBIANA: 2002, C-578) Como se observa, el organismo cuando asuma la competencia en un caso particular, y después de culminada la actuación tenga a su cargo la fijación de la sanción, podría válidamente hacer uso de estas cláusulas aun frente a la prohibición de derecho interno.

Pues bien determinados estos elementos preliminares, se avizora una tendencia de indeterminación frente al non bis in ídem, que es preciso abordar en concreto desde el punto de vista material con base en los elementos que hacen parte de la discusión.

\subsection{El non bis in ídem material}

En cuanto a este primer aspecto, en el sistema continental, la doctrina y la jurisprudencia han reiterado la necesidad de constatación de la unidad de contenido del injusto frente a la identidad de la infracción, en el requisito de la triple identidad de sujetos, hechos y fundamento, sin embargo la constatación de la unidad delictiva del non bis in ídem, es una consecuencia a la que se llega luego de tomar en cuenta las reglas de la especialidad, subsidiariedad y consunción. (GONZALEZ \& GORRIZ: 2003: 242)

Frente a este punto, es preciso advertir que en los elementos del crimen del ER, concretamente en la regla $\mathrm{N}^{\circ} 9$ de la introducción, se establece en términos sencillos que: ".... Una determinada conductapuedeconfigurarunoomáscrímenes...". Lo cual puede indicar, que en el Estatuto, se podrá acudir más que a la determinación de las conductas bajo las reglas anotadas (consunción especialidad y subsidiariedad), a la sumatoria de todas las que han sido ejecutadas por un sujeto activo en particular. Este punto parece contrariar en principio el artículo 31 del Código Penal, que establece las reglas del concurso de Conductas punibles en el derecho nacional, en la medida en que el argumento de la regla $9^{\mathrm{a}} \mathrm{de}$ los elementos del crimen, aparece bajo criterios indeterminados y flexibles, frente a la concreción del derecho interno.

Si acudimos a los casos actuales del organismo internacional, se observa, por ejemplo en la orden de detención librada en contra de Ahmad Muhamad Harun de 27 de abril de 2007, librada en el caso Darfur (Sudán) por parte de la CPI, que en la misma, se realiza la acumulación de 51 cargos relativos a conductas que indistintamente corresponden a lesa humanidad y crímenes de guerra, más que a su determinación en un concurso material específico ${ }^{3}$. Esta decisión sin duda obedece a una valoración distinta de la conducta, si la vemos con la perspectiva del derecho continental.

Tanto así que en el texto de la orden referida, no se analiza la concurrencia de las mismas, sino que únicamente se presentan los hechos que corresponden a determinado crimen, lo cual es

3 DOCUMENTO ICC-02-05-01-07 De 27 de abril de 2007. Orden de detención librada por la Sala de Cuestiones Preliminares. Situación Darfur Sudán- En el caso AHMAD MUHAMAD HARUN. Disponible En http://www.icc-cpi.int/iccdocs/doc/doc345893.PDF (Consultada el día 5.03.2009) 
suficiente para motivar la orden de detención (Doc. ICC-02-05-01-07 de 27.04.2007. ICCCPI. 2009) ${ }^{4}$. Como se puede ver, en la práctica, los Magistrados acuden a reglas más sencillas que las descritas por el sistema continental, sin embargo, es claro que el planteamiento sobre la conducta, en el ejemplo citado, obedece a unos hechos que corresponden a unos elementos del delito. Es decir, se hace una mínima sustentación fáctica y jurídica sobre el crimen en el cargo respectivo, situación que es relevante desde el punto de vista del principio de legalidad y por supuesto del non bis in ídem material. A continuación se verá en específico, cada uno de los fenómenos avisorados en forma preliminar en casos específicos.

\subsubsection{Concurrencia de leyes}

En el primer caso de concurrencia relativa a la especialidad, la aplicación de una ley que es especial, prevalece sobre otra de contenido más general, por cuanto queda ya contenida en aquella. En este caso la ley especial se aplicará de preferencia a la general. (GONZALEZ \& GORRIZ: 2003: 242) En la subsidiariedad, el precepto subsidiario se aplica en defecto del tipo principal. Es un tipo de recogida, en donde, suele exigirse una patente de afinidad entre los tipos principal y subsidiario. (GONZALEZ \& GORRIZ: 2003: 243) En cuanto a la consunción, este principio interviene cuando "...un tipo penal determinado, absorbiendo en sí el desvalor de otro, excluye a éste de su función punitiva...". (GONZALEZ \& GORRIZ: 2003: 243) En definitiva, en ausencia de estos criterios en el art. 20 E.R., sería deseable que el intérprete aplique el non bis in ídem desde este punto de vista, para determinar la existencia de una sola conducta en la concurrencia aparente de delitos.

En ese orden de ideas, se debe apelar a las reglas que en nuestro sistema corresponde a los

4 DOCUMENTO ICC-02-05-01-07 De 27 de abril de 2007. Orden de detención librada por la Sala de Cuestiones Preliminares. Situación Darfur Sudán- En el caso AHMAD MUHAMAD HARUN. Disponible En http://www.icc-cpi.int/iccdocs/doc/doc345893.PDF (Consultada el día 5.03.2009) principios analizados (Art. 31 Código penal), los cuales no aparecen regulados en otros sistemas jurídicos y menos en el ER. (GONZALEZ \& GORRIZ: 2003: 247) Estas reglas tampoco aparecen en las decisiones de la CPI que pueden ser examinadas en la actualidad y por el contrario, lo que más se denota es su independencia y autonomía en el punto, mucho más prevalente hacia el análisis anglosajón ${ }^{5}$.

Lo anterior, tiene fundamento no sólo en la expresión de la regla $9^{a}$ de los Elementos del Crimen advertida anteriormente, señalando que una conducta puede configurar uno o más crímenes, sino también en el orden de prelación de aplicación de las normas que dispone la Corte, (COSTTI \& FRONZA: 2009: 108-109) según las cuales: en primer lugar se debe aplicar el Estatuto de Roma, los elementos del Crimen y las reglas de procedimiento y prueba; en segundo lugar, los tratados y los principios de derecho internacional aplicables, incluidos los principios de derecho internacional aplicables a los conflictos armados. En su defecto, los principios generales del derecho que derive la Corte de los sistemas jurídicos del mundo cuando procedan o el derecho interno de los Estados que normalmente ejercerían jurisdicción sobre el crimen, siempre que estas normas no sean incompatibles con el Estatuto, ni con el Derecho Internacional, ni con las normas y principios internacionalmente reconocidos (Art. 21 ER). (ANELLO: 2003: 61)

De acuerdo con esta norma, la interpretación sugerida por los autores González Cussac y Gorriz Royo, no parece tener en principio

5 Esto Sin duda puede ser observado en las siguientes decisiones: Documento ICC-02-05-01-07 De 27 de abril de 2007. Orden de detención librada por la Sala de Cuestiones Preliminares. Situación Darfur Sudán- En el caso AHMAD MUHAMAD HARUN. Disponible En http://www.icc-cpi.int/iccdocs/doc/doc345893.PDF (Consultada el día 5.03.2009) - Documento ICC-02-05-01-09 de 04 de marzo de 2009. Caso de OMAR HASSAN AHMAD AL BASHIR. Orden de detención librada por la Sala de Cuestiones Preliminares. Situación Darfur Sudán- Disponible en http://www.icc-cpi.int/iccdocs/doc/ doc644487.pdf (Consultada el día 05.05.2009). 
aplicación, si resulta incompatible con las reglas primarias de interpretación del ER. que remiten inicialmente, la interpretación al propio Estatuto, a los elementos del crimen y a las reglas de procedimiento y prueba. E incluso de acuerdo con lo preceptuado en tales preceptos, se puede recurrir a las normas internas por vía residual a la fuente interna sólo en caso de lagunas o donde las fuentes principales y secundarias se revelen inadecuadas para analizar un caso concreto. (COSTTI \& FRONZA: 2009: 108-109).

\subsubsection{Concurrencia de delitos}

Tradicionalmente en el sistema continental, el concurso de infracciones se presenta cuando un mismo sujeto ha violado varias veces la ley penal y por lo tanto ha de responder por varios delitos, lo que se configura por cuatro requisitos esenciales: a) pluralidad de infracciones; b) Unidad o pluralidad del objeto valorado que da lugar a las dos formas de concurso ideal (formal) y real (material); c) Unidad de Sujeto; d) Unidad de enjuiciamiento ${ }^{6}$.

En la normativa colombiana las reglas del concurso de conductas punibles aparecen descritas en el art. 31 del C.P. ${ }^{7}$ En el caso del Estatuto de Roma de 1998, el criterio parece inclinarse por una discrecionalidad judicial, sin que aparezcan fundamentos normativos definitivos. Este evento parece determinarse en la pena, conforme a lo

6 El tema ha sido objeto de tratamiento por muchos autores en el derecho Colombiano, toda vez que hace parte de nuestra tradición jurídica nacional en materia penal y coincide con los principios esbozados en el presente aparte. CFR. SOLORZANO GARAVITO Carlos Roberto. Unidad y Pluralidad de Acción, el Concurso de Conductas Punibles. Primera Edición, Ediciones Nueva Jurídica, Bogotá D.C., enero de 2002. Pp. 195 a 211. FERRE OLIVE Juan Carlos Et. Al. Derecho Penal Colombiano, Parte General. Primera Edición, Grupo Editorial Ibañez. Bogotà D.C., 2010. Pp. 569 a 589. VELASQUEZ VELASQUEZ Fernando. Manual de Derecho Penal Parte General. Ed. Temis, Bogotá D.C. 2002. Pp. 467 a 580.

7 Los elementos del concurso han sido tratados con propiedad en la siguiente decisión: CFR. CORTE SUPREMA DE JUSTICIA, Sala de Casación Penal. Sentencia de 25 de julio de 2007. Proceso 27383. MP. Yesid Ramírez Bastidas. establecido en el art. 78. ER., según lo especificado en su numeral $3^{\circ}$ que señala:

(...)Cuando una persona haya sido declarada culpable de más de un crimen, la Corte Impondrá una pena para cada uno de ellos y una pena común en la que se especifique la duración total de la reclusión. La pena no será inferior a la más alta de cada una de las penas impuestas y no excederá de 30 años de reclusión o de una pena de reclusión a perpetuidad de conformidad con el párrafo 1 b) del artículo 77 (...)

Este concepto sigue la tradición según González Cussac y Gorriz Royo de los demás tribunales internacionales ad hoc precedentes a la CPI, que tampoco pudieron articular un criterio unánime para los casos de concursos de normas y de delitos analizados. (GONZALEZ \& GORRIZ: 2003: 254) Los criterios establecidos según esta postura, surgieron a raíz de la inquietud de si se podía perseguir y condenar por determinada conducta de un mismo sujeto, por un crimen contra la humanidad y a la vez por un crimen de guerra $^{8}$. O bien condenar por un crimen de lesa humanidad y además de genocidio, por una sola conducta cometida por el mismo sujeto. (GONZALEZ \& GORRIZ: 2003: 254)

En casos concretos como el juicio a Tadic (1999) se estableció el criterio de cumplir de manera simultánea las penas impuestas por cada cargo en la condena, además de proponer diferencias de gravedad entre delitos de lesa humanidad $y$ de guerra. En concreto, el Tribunal para la antigua Yugoeslavia, finalizó en este caso (Tadic - 1999), con una sentencia en la que se afrontó el problema de individualizar los delitos asignando penas diferentes por cada una de las

8 En el caso Kunarac y Kovac, Decision on the form of the indictment, 4 noviembre de 1999 (IT-96-23-PT): Se permite que se acumulen diferentes delitos para los mismos hechos; la acumulación jurídica sólo es relevante para la sentencia. AMBOS Kai, Derecho y Proceso Penal Internacional. Ensayos Críticos. Ed. Distribuciones Fontamara. Primera Edición. Mexico D.F. 2008. P. 190 
acusaciones o cargos ${ }^{9}$. En concreto Tadic fue condenado por 11 cargos, 6 de ellos relativos a crímenes contra la humanidad y a 5 violaciones de las leyes y costumbres de la guerra, con diversas penas de prisión $(6,7,6,7,6,10,9$ y 20 años). (GONZALEZ \& GORRIZ: 2003: 255)

En esta resolución el Tribunal referido ${ }^{10}$ estableció una pena final, pero sin que el mismo se pronunciara sobre algún tipo de concurrencia de conductas que debía apreciarse entre las distintas acusaciones. Para tal efecto, dicha instancia estableció que cada una de las penas fuera cumplida en forma simultánea entre sí (GONZALEZ \& GORRIZ: 2003: 255) con una sumatoria total de 20 años de prisión, por los crímenes arriba señalados, luego de las apelaciones presentadas a la Condena Iniciall ${ }^{11}$.

De igual forma, en los casos Clement Kayishema y Obed Ruzinda, se hace necesario destacar el empleo de un "test" para detectar la concurrencia de delitos, usado anteriormente en el caso Prosecutor vs Akayesu (Tribunal de Ruanda), el cual advierte que sólo es posible condenar a un acusado de dos o más delitos en relación al mismo grupo de hechos, en las siguientes circunstancias: 1) cuando los delitos contengan diferentes elementos; o (2) cuando los preceptos

9 En el caso Dusko Tadic, toda la información relativa al juicio sentencias y apelaciones que fueron impuestas, se observa la consideración independiente de los crímenes sin recurrir a las reglas concursales propuestas por Gonzàlez Cussac y Gorriz Royo. Por el contrario se analizan los hechos y se consideran las pruebas en cada evento. Para ampliar dicha información favor consultar; Tadić (IT-94-1) "Prijedor". Case Information Sheet. Disponible en: www.icty.org/x/cases/tadic/cis/en/cis_tadic_en.pdf

10 Los diferentes procesos adelantados ante el Tribunal Internacional para la Antigua Yugoeslavia se pueden consultar en la siguiente dirección: www.icty.org/action/cases/4

11 En la sentencia de juzgamiento y apelación del caso Dusko Tadic se definió la sentencia definitiva a cumplir de 20 años. La misma se puede consultar en el siguiente documento: International Tribunal for the Prosecution of Persons Responsible for Serious Violations of International Humanitarian Law Committed in the Territory of the Former Yugoslavia since 1991Case No.: IT-94-1-A and IT94-1-Abis Date: 26 January 2000. Disponible en: www. icty.org/x/cases/tadic/acjug/en/tad-asj000126e.pdf. sometidos a consideración, protejan intereses sociales diferentes, o (3) cuando sea necesario formular una condena a través de ambos delitos con la finalidad de reflejar por completo lo que cometió el acusado. (GONZALEZ \& GORRIZ: 2003: 258)

En definitiva, en la jurisprudencia penal internacional, se ha admitido condenar un grupo de ilícitos, por más de un delito sobre la base de apreciar: a) la no reiteración de elementos típicos; b) la afección a diferentes intereses sociales y/o la inexistencia de infracciones absorbentes que consuman injustos menores "lex consumens". (GONZALEZ \& GORRIZ: 2003: 260) Por otro lado, en los casos en que los tribunales han impuesto penas múltiples, o conjuntas, su cumplimiento ha sido dispuesto de manera simultánea (cumplimiento de pena más común) y nunca sucesiva, es decir, no se ha dispuesto hasta el momento, el cumplimiento de una pena tras otra, en un tribunal internacional. Igualmente, la cuantificación de la pena a cumplir se ha determinado a partir de la pena de más larga duración, la que determina el tiempo real a cumplir. (GONZALEZ \& GORRIZ: 2003: 260) Esta solución se puede asimilar al concurso ideal del derecho penal continental ${ }^{12}$.

Por último, en algunos casos, haciendo uso del poder discrecional, los tribunales internacionales han optado por imponer varias penas bajo la forma de una pena conjunta, incrementando el castigo más grave de los impuestos al condenado. Este aspecto se asemeja según los tratadistas referenciados (González C. y Gorriz R.), a la figura del concurso real del derecho continental. (GONZALEZ \& GORRIZ: 2003: 260) En este sentido, el sistema que parece erigirse en la CPI, se adecúa al estándar de juicio justo que permite acercar las tendencias $y$ convertir la actuación en un proceso mixto que pueda encontrar acercamientos entre el derecho

12 Como se mencionó atrás, en los Tribunales Internacionales, la posibilidad de Acumulación de cargos, parece posible sólo en la fijación de Sentencia. AMBOS KAI. Ob. Cit. P. 190 
continental y el Common Law. (AMBOS: 2008: 220) Sin embargo, es conveniente señalar que no es tan fácil pronosticar una postura como la expuesta por los autores referidos, toda vez que la tendencia que se observa en la CPI, es la creación de un sistema propio que puede adicionar aspectos del derecho continental.

En este sentido, en principio las reglas del ER., tienen una orientación más cercana al Common Law, pero seguramente, con la intervención de distintas causas procedentes de diversos sistemas jurídicos del mundo, la búsqueda de principios comunes entre los mismos, va a ser cada vez más frecuente y la mixtura entre diversas tendencias cada vez mayor. (AMBOS: 2008: 220-221) En todo caso, se observa inicialmente, la tradición asumida desde los tribunales ad hoc, tal y como se plantea en las órdenes de arresto libradas, como las relativas al caso de Ahmad Muhammad Harun (27.04.2009) y Omar Hassan Ahmad Al Bashir (4.03.2009) - Caso Darfur-Sudán (Doc. ICC-02-05-01-09 de 04 de marzo de 2009 ICC-CPI, 2009. $)^{13}$.

Sobre este punto, la tendencia en los casos asumidos por la CPI como se puede ver anteriormente, se orienta a dar prevalencia a las reglas del Estatuto, independientemente del sistema jurídico de que se trate, con lo cual, es probable que estas reglas de unidad de acción pasen a un segundo plano en el sistema internacional del ER. y por ende, la tradición continental, deba ceder a una tradición anglosajona en el seno de las actuaciones de la CPI. Precisamente esta postura no sólo se deriva del contenido de la regla $9^{a}$ contenida en los elementos del Crimen ${ }^{14}$, sino también, en virtud de la interpretación con respecto a la

13 Disponibles en http://www.icc-cpi.int/iccdocs/doc/ doc345893.PDF (Consultada el día 5.03.2009) -Y http://www.icc-cpi.int/iccdocs/doc/doc644487.pdf (Consultada el día 05.05.2009).

14 Los elementos del Crimen en el derecho interno, fueron declarados excequibles mediante la siguiente decisión: CORTE CONSTITUCIONAL, Sentencia C-801 de 10 de noviembre de 2009. MP. Gabriel Eduardo Mendoza Martelo. aplicación del art. 21 ER., toda vez que existe un orden de prevalencia de normas en donde el sistema interno aparece como norma terciaria de interpretación, con carácter ambiguo, en la medida en que puede ser examinada mientras no sea incompatible con el tratado de Roma. (SCHABAS: 1999: 277-278) A pesar de esta postura que parece tener un carácter imperante, autores como Kai Ambos sostienen que de todas formas, la influencia continental puede verse en el intercambio probatorio del sistema de la CPI, toda vez que es posible que en el plano interestatal se deban realizar interpretaciones tendientes a verificar aspectos investigativos de causas adelantadas ante el organismo supranacional. (AMBOS: 2008: 220-223) Ello sin duda puede implicar una consulta imprescindible de fuentes terciarias y una obligada mezcla de interpretaciones, que puedan dar paso al sistema interno y a posturas dogmáticas como la analizada.

\section{PAUTAS ESPECÍFICAS EN RELACIÓN CON LOS CONCURSOS ENTRE LESA HUMANIDAD, GENOCIDIO Y CRÍMENES DE GUERRA}

Pues bien, en concreto desde el factor de competencia de la CPI, atendiendo la ausencia de criterios sobre las situaciones y reglas del concurso de conductas punibles analizadas, conviene precisar que el bien jurídico en particular, en el crimen de lesa humanidad (art.7 E.R.), se manifiesta en forma individual, frente a bienes jurídicos individuales como la vida, la integridad física, libertad sexual etc., cuya titularidad corresponde a sujetos pasivos individuales. Este bien jurídico individual también se precisa en los crímenes de guerra (art. 8 E.R.). Por el contrario, los atentados en los crímenes de genocidio se diferencian de los atentados de lesa humanidad por tratarse de conductas que afectan un bien jurídico colectivo o supraindividual. (GONZALEZ \& GORRIZ: 2003: 262)

En esta medida, en la persecución de atentados de lesa humanidad, cada una de las agresiones perpetradas contraunodelosbienesindividuales 
de los descritos en el art. $7^{\circ}$ E.R., constituiría un crimen de lesa humanidad, al igual que los atentados de guerra, frente a las infracciones graves de los Convenios de Ginebra y demás legislación complementaria, descrita en el art. $8^{\circ}$ E.R. Por su parte, la comisión durante un período de tiempo de varias de las modalidades previstas en el art. $6^{\circ}$, corresponden a crímenes de Genocidio. Bajo este criterio, se plantea (GONZALEZ \& GORRIZ: 2003: 264) que en el caso de concurrencia entre conductas de lesa humanidad con el genocidio, es posible la presencia de un concurso ideal. Esta misma consecuencia se desarrolla para la concurrencia entre genocidio y crímenes de guerra, de tal modo que los atentados, constituyen a la vez dos categorías de crímenes distintos, con lo cual no aparece contrariedad con la postura anglosajona analizada.

Sobre este punto González Cussac y Gorriz Royo advierten, que ello podría suceder, si mediante una misma conducta se comete un delito de genocidio y a la vez, varias modalidades de lesa humanidad y que esto podría darse también, en el caso de concurrencia entre modalidades totalmente diversas de crímenes contra la humanidad y crímenes de guerra, lo cual, debería dar lugar a la aplicación de reglas similares al concurso real. (GONZALEZ \& GORRIZ: 2003: 264) En todo caso es necesario precisar, conforme lo plantea Carolina Anello, que en los Crímenes de Guerra a diferencia de las conductas de lesa humanidad, no se exige elementos de masividad o sistematicidad, lo cual puede incorporar criterios distintivos de tales crímenes. (ANELLO: 2003: 56) De igual forma, en las órdenes aludidas anteriormente, libradas por la CPI, lo único que se observa es un listado de delitos sin un análisis que concuerde con la perspectiva propuesta por González Cussac y Gorriz Royo, analizados anteriormente $^{15}$. Al respecto, es posible que

15 Documento ICC-02-05-01-07 De 27 de abril de 2007. Orden de detención librada por la Sala de Cuestiones Preliminares. Situación Darfur Sudán- En el caso AHMAD MUHAMAD HARUN. Disponible En http://www.icc-cpi.int/iccdocs/doc/doc345893.PDF el elemento de intencionalidad del genocidio implica la destrucción total o parcial de un grupo determinado, mientras que en lesa humanidad estos actos deben contar con los actos sistemáticos y generalizados. En el fondo, es posible que al adicionar a los actos sistemáticos y generalizados, contra una población civil, la firme intención de la destrucción de un grupo determinado, esto sería suficiente para decidir en torno al concurso ideal con la correspondiente subsunción en torno al Genocidio.

Ahora bien, en cuanto a las múltiples relaciones concursales entre las conductas de lesa humanidad y los demás crímenes, en primer lugar, resulta de difícil apreciación, la relación con respecto al genocidio, sin embargo en el Estatuto de Roma (art. $6^{\circ}$ ), el elemento subjetivo establecido (mens rea), es el factor determinante, por cuanto, el mismo que responde a la intención de destruir total o parcialmente un determinado grupo, no aparece desarrollado en las conductas de lesa humanidad.

Aplicando estas consideraciones a los delitos $6^{\circ}$ y $7^{\circ}$ E.R., es posible aseverar que en esta relación, es muy difícil articular la especialidad o subisidiariedad, atendiendo las diferencias anotadas y por lo tanto en una situación particular como por ejemplo en un acto consistente en cometer "matanza de miembros del grupo" (art. 6. a. E.R.). y "asesinato" (art. 7.1.a. E.R.), habrá que atender el elemento subjetivo reseñado para resolver la situación. (GONZALEZ \& GORRIZ: 2003: 265) Por el contrario si aparece el elemento subjetivo del injusto de destrucción del grupo, junto con el asesinato como parte de un ataque sistemático y generalizado contra una población civil, el asunto parece encajar dentro del concurso ideal, que supone la lesión al bien jurídico "vida", (GONZALEZ \& GORRIZ: 2003:

(Consultada el día 5.03.2009) - Documento ICC-0205-01-09 de 04 de marzo de 2009. Caso de OMAR HASSAN AHMAD AL BASHIR. Orden de detención librada por la Sala de Cuestiones Preliminares. Situación Darfur Sudán- Disponible en http://www. icc-cpi.int/iccdocs/doc/doc644487.pdf (Consultada el día 05.05.2009) 
265) y las modalidades concurren tanto en Genocidio como en lesa humanidad.

En segundo término, en cuanto a los crímenes contra la humanidad y los crímenes de guerra, según González C. y Gorriz R., se pueden presentar conflictos en cuanto a las conductas relacionadas con ataques a la libertad sexual de concretos individuos. (GONZALEZ \& GORRIZ: 2003: 266) Por ejemplo: las conductas previstas en el art. 7.1.g. E.R. y las descritas en él, como crímenes de guerra en el art. 8.2. b) xxii) E.R. Y a su vez, cualquiera de las anteriores modalidades de conducta pueden concurrir con la modalidad del crimen de guerra prevista en el apartado 8.2.e) vi. E.R.

Según los autores analizados, estas relaciones que se pueden multiplicar en el Estatuto, corresponden a una deficiente técnica legislativa en la que la nota predominante es la reiteración en la descripción de las conductas y las remisiones en los propios preceptos por ejemplo: $\mathrm{El}$ artículo 8.2. b. xxii E.R. remite a la definición de embarazo forzado del art. 7.2.f) E.R. Pues bien, según estos autores, tratándose de idéntico bien jurídico, lo adecuado es buscar criterios de especialidad, (GONZALEZ \& GORRIZ: 2003: 267) sin embargo, esta postura puede ser viable, si se tratara de un caso que involucre ante la $\mathrm{CPI}$, nuestro sistema jurídico. E incluso, para un caso como este, de acuerdo con las definiciones de los elementos del crimen, un criterio adecuado para saldar esta discusión, reside en los elementos comunes para cada conducta, acudiendo a nociones como por ejemplo, las descritas en el derecho interno colombiano para Crímenes contra el DIH y personas protegidas (Titulo II Ley 599 de 2000), relacionadas con el elemento "con ocasión y en desarrollo de un conflicto armado (internacional - no internacional", para diferenciar el alcance de lesa humanidad y los crímenes de guerra. (CORTE: 2007, C-291)

Otro asunto controvertido, se puede presentar en el caso del asesinato (art. 7.1.a.) E.R. y del crimen de guerra, consistente en matar intencionadamente (art. 8.2.a.i. E.R.). Este caso parece acomodarse a un concurso aparente, que puede resolverse bajo criterios de especialidad, toda vez que el asunto, una vez contrastado con los ingredientes relacionados con las personas y bienes protegidos por el DIH, sería suficiente para determinar la producción de una sola conducta, es decir, la perteneciente al crimen de guerra. (GONZALEZ \& GORRIZ: 2003: 267) En nuestro sistema, podríamos válidamente hacer la diferencia, bajo los elementos con ocasión y en desarrollo de conflicto armado reseñados anteriormente. En el mismo sentido se puede acoger este principio para la tortura de lesa humanidad (art. 7.1.f. E.R.) y la tortura como crimen de guerra (art. 8.2. a, ii E.R.).

Sin embargo, en los casos concretos que pueden tener lugar en la realidad nacional, la postura de los autores analizados no ofrece una fácil solución, dados los límites que entre uno y otro comportamiento (Crímenes de GuerraCrímenes de Lesa Humanidad) parecen tener en la vida real. Por lo menos en la situación colombiana, como por ejemplo, en los hechos denunciados por "El Tiempo", en su edición del 2 de marzo de 2009, relativos a la violencia sexual contra las mujeres con ocasión del conflicto armado, se relata el asesinato de parte de las FARC, el 19 de agosto del 2006 de una joven de 15 años, por haber sostenido una relación sentimental con un policía. (EL TIEMPO: 2009, marzo) Como se puede ver, en este tipo de actos coinciden diversos crímenes que no solamente concurren en una de las conductas como por ejemplo la tortura, toda vez que el cuerpo de la menor fue hallado días después con signos de violencia física.

Así mismo, la problemática de resolver este caso, se centra en que tipo de elementos son necesarios, para determinar si la relación con el policía obedecía a un plan sistemático de eliminar a todos aquellos que sean amigos o colaboradores con el adversario o si esto se relaciona con mayor vigor, con el Conflicto Armado Interno y tiene que ver con los atentados en persona protegida, o en últimas si tiene que ver con los dos motivaciones de la conducta. 
Sobre esto, es posible que en caso de duda sobre el alcance del elemento relacionado con el conflicto armado, la situación deba resolverse a favor del crimen de lesa humanidad. Ahora, si concurren las dos motivaciones (Guerra - Lesa Humanidad), para efectos de reprimirlas simultáneamente en un caso específico, siguiendo la orientación que se muestra en las decisiones de la CPI, válidamente se puede aceptar desde la mirada continental, la tesis de un concurso ideal de conductas, y en la tendencia anglosajona, también se puede determinar la comisión de dos crímenes diferenciados por cada motivación ${ }^{16}$. Lo anterior implica en síntesis, que pese a las interpretaciones de derecho continental advertidas que es posible desde los factores de legalidad y non bis in ídem examinados, que la tendencia del sistema concebido en el organismo internacional, eventualmente atienda las pautas establecidas en los tribunales internacionales anteriores a su establecimiento, que procuran la creación de un derecho autónomo mixto con una tendencia hacia el common law, lo cual parece confirmarse en las cláusulas descritas, así como en los primeros ejemplos citados.

Así mismo, frente al derecho interno, los principios derivados de los arts. 28 y 29 de la Const. Política, $6^{\circ}, 8^{\circ}, 31,34$ y ss. del C.P. servirán de fundamento adicional para complementar el vacío normativo que se aprecia en el Estatuto de Roma, dada su ineludible perspectiva orientada hacia common law. Todo ello en la medida en que sean atendibles tales presupuestos en el seno del organismo internacional.

En último término, las múltiples relaciones que se presentan entre las conductas de lesa humanidad, genocidio y los crímenes de guerra, advierten la necesidad de desarrollo jurisprudencial en torno a criterios unificados para la solución de los problemas de concurrencia, sobre todo para aquellos sistemas que no corresponden a la tendencia anglosajona

16 Documento- ICC-01/04-01/06-2-tEN 03-04-2006 2/5 UM PT - Warrant of Arrest. The Prosecutor Vs Thomas Lubanga Dyilo. Disponible en: www.icc-cpi.int/iccdocs/ doc/doc191959.PDF. Consultada el 17.10.10 observada en el presente documento, màxime frente a los bienes jurídicos internacionales que son objeto de lesión.

\section{SITUACIONES PROCESALES - PRINCIPIO DE LEGALIDAD, NON BIS IN ÍDEM PROCESAL Y COMPETENCIA.}

Finalmente, todas estas consecuencias terminan en el plano procesal, toda vez que uno de los terrenos que más alcance ha tenido en el derecho colombiano, se centra en las consecuencias procesales del non bis in ídem, en sus componentes descritos en el artículo $8^{\circ}$ del C.P. (ley 599 de 2000), estatuto en el que se determina, la prohibición de doble juzgamiento, salvo lo establecido en los instrumentos internacionales.

Este concepto se inscribe en relación con la cosa juzgada en términos de su relativización, en virtud de la posibilidad de convocar a una nueva oportunidad procesal, instancias ya falladas, bajo criterios que residen en la acción de revisión del proceso ${ }^{17}$, tal y como ha sido sostenido por la Corte Constitucional en sucesivas decisiones que confirman esta teoría. (CORTE: 2003, C-004) Pues bien uno de estos factores, se erige en el aspecto procesal, en un primer momento en las circunstancias que motivan la Competencia en el ER., conforme a los condicionamientos establecidos tanto en el artículo 17 E, $R$, como a raíz de las consecuencias descritas en el art. 20 E.R.

El ejercicio de la competencia de la CPI, se desarrolla con ocasión de situaciones que provoquen la impunidad en el derecho interno,

17 Este punto se puede verificar, cuando una sentencia es producto de una investigación que no ha comprendido "...todos los hechos, todos los autores y todas las circunstancias determinantes del hecho de forma seria e imparcial, incumpliendo los parámetros establecidos por el debido proceso del derecho internacional..." Es decir, no existen criterios de interés de Justicia Material. CFR. PENAGOS TRUJILLO Sandra Cristina y SANCHEZ POSSO Juan Carlos. El non bis in ídem y la Cosa Juzgada en el Estatuto de Roma de la Corte Penal Internacional. Grupo Editorial Ibáñez. Bogotà D.C. 2007. P. 177. 
que desconozcan tanto las garantías procesales como la justicia material o efectiva en el Estado Parte, conforme a lo regulado en los artículos 17 y 20 E.R. Sólo ante tales situaciones, la proscripción del non bis in ídem es reemplazada por la posibilidad de un nuevo juzgamiento ante el organismo internacional, sobre la base de unos hechos que corresponden de manera indefectible a unos crímenes que son parte de su competencia (art. $5^{\circ}$ E.R.) y no simplemente sobre situaciones de hecho, con lo cual se reitera el contenido material del non bis in ídem en el ámbito de la determinación (nullum crimen sine lege) y en los aspectos relacionados con las relaciones concursales de las conductas de la CPI.

Desde este punto de vista, la valoración de los hechos frente a tales conductas, establece la relación entre la determinación de la conducta, su investigación, su adecuación típica y fundamento, con lo cual se cumple con la orientación de nuestro derecho interno de identidad entre el sujeto, el hecho y el fundamento jurídico (Arts. 6 y 8 C.P. Y 29 Const. Pol).

Este fenómeno se observa con mayor precisión en situaciones, no sólo relacionadas con la admisión de la actuación (art. 17 E.R.) sino también en circunstancias propias del ejercicio de la investigación en las cuales se reitera que el Fiscal, al decidir si ha de iniciar una investigación, tendrá en cuenta si la información de que dispone constituye fundamento razonable para creer que se ha cometido o se está cometiendo un crimen de la Corte (literal a, Art. 53 E.R.), es decir debe analizar el contenido fáctico de los hechos y si corresponden a un crimen de competencia de la CPI, con lo cual, se expresan los dos ámbitos analizados del non bis in ídem, tanto en su aspecto material como procesal.

Finalmente, es preciso manifestar que la orientación en general del Estatuto, reside en la consideración de estos dos aspectos, tanto de orden sustancial como material; tanto en la evaluación de los hechos y su adecuación a los crímenes de su competencia, ámbito que de igual forma se reitera en los requisitos de la orden de detención (Literal a) Art 58 E.R.; en la confirmación de los cargos, art. 61, en particular en el numeral $5^{\circ}$ y en general en todo el contexto de la norma referida. Por su puesto también, en los ámbitos del juicio, la sentencia y demás actuaciones posteriores.

\section{CONCLUSIONES}

La relación legalidad, non bis in ídem material, permite observar que la tendencia continental que aboga por determinar la responsabilidad por una sola conducta que resulte asociada a la comisión de un crimen de la competencia de la CPI en las relaciones concursales, tiene innumerales aspectos que la hacen una tendencia residual y no principal para los fines del organismo internacional, sin que ello signifique que es la tendencia absoluta del instrumento de Roma.

Así mismo, es viable aseverar que la tendencia anglosajona del instrumento, en principio, no parece conciliar este ámbito y se orienta más hacia la sumatoria de conductas para el ejercicio de su competencia,(se encuentren relacionadas o no las diversas tipicidades). En torno a este aspecto, en el derecho interno, debido a la orientación continental del sistema nacional, se adicionan insumos relacionados con las reglas de la concurrencia de delitos perfectamente atendibles en la competencia del tribunal internacional analizado (Art. 31 Ley 599 de 2000), (APONTE: 2003: 234) en dado caso que fuere discutido este ámbito material del non bis in ídem, pese a que de todas formas, la tendencia de la CPI parece ser propia y autónoma, aun frente a principios de raigambre continental como los analizados.

Igualmente, las múltiples relaciones que se desarrollan entre las conductas analizadas, concretamente de lesa humanidad, permiten avisorar un bien jurídico de mayor entidad diversa a los descritos en el derecho interno, todavía sin desarrollo normativo actual, lo cual puede ser objeto de discusión frente al derecho interno, sin que aun se puedan establecer 
los alcances de esta determinación ${ }^{18}$. En este sentido, es viable plantear un nuevo desarrollo legal en torno a los delitos contra la comunidad internacional que podría agrupar además de las conductas de lesa humanidad, la conducta de genocidio y los crímenes de guerra, para evitar esta confrontación -derecho interno, Estatuto de Roma-, en la que al final prevalezca la norma internacional, en sacrificio de la interna.

En definitiva, la perspectiva planteada permite identificar una progresividad normativa pendiente, que no sólo puede ser agotada con interpretaciones jurisprudenciales a nivel interno, sino que implican una mejor correspondencia entre el derecho penal internacional planteado en el ER. y las normas internas de los delitos y las penas, con el fin de salvaguardar los futuros inconvenientes originados precisamente de la aplicación y admisibilidad de un asunto nacional ante la $\mathrm{CPI}$, en perjuicio de la cosa juzgada interna.

Finalmente, siendo esto así, la valoración que exige el non bis in ídem, para una situación en particular en que sus límites deban ser rebasados, por las razones anotadas, no pueden prescindir de su examen material, consistente en la relación entre los hechos y la consecuencia jurídica que reposa en una conducta punible, para lo cual es imprescindible el examen del nullum crimen sine lege del Estatuto (Art. 22 ER) y las circunstancias tanto jurídicas, como sociales e incluso políticas que impidan el recurso efectivo y la justicia material, conforme a lo establecido en el art. 20 E.R. Una valoración en sentido contrario resultaría incompleta para los fines señalados y además no contaría con

18 En todo caso para algunos, el desarrollo de la ley 1268 y la declaratoria de excequibilidad por medio de la sentencia C-801 de 2009, mediante la cual se determinò la correspondencia en el derecho interno de los elementos del crimen y las reglas de procedimiento y prueba de la CPI, parecería que da término a esta discusión, sin embargo, estos instrumentos deben ser entendidos en el ámbito de vigencia del Estatuto de Roma de 1998, conforme a la Sentencia C-578 de 2002, con lo cual el desarrollo interno de los comportamientos de lesa humanidad seguiría pendiente. innumerables argumentos que aparecen descritos en las cláusulas del Estatuto de Roma.

\section{BIBLIOGRAFÍA}

\section{Doctrina}

AMBOS, K. (2008). Derecho y Proceso Penal Internacional, Ensayos Crítico (1ra Ed.). Ciudad de México, México: Distribuciones Fontamara.

(ANELLO, C.S. (2003). Corte Penal Internacional. Buenos Aires, Argentina: Editorial Universidad.

APONTE, A. (2003). Persecución penal nacional de crímenes Internacionales en América Latina y España. Montevideo, Uruguay: Editorial Fundación Konrad Adenauer Stiftung, Instituto Max Planck.

COSTTI, M. \& FRONZA, E. (2009). Capítulo

II: Las Fuentes. (Y, VIVEROS, Trad.).

Introducción al Derecho Penal Internacional. Bogotá, Colombia: Editorial Kimpres-Universidad Libre, Bogotá 2009

FERRE, J. C. (2010). Et. Al. Derecho Penal Colombiano, Parte General (1ra Ed.). Bogotá, Colombia: Grupo Editorial Ibañez.

FRONZA, E. (2009). Las Sanciones. Introducción al Derecho Penal Internacional. (Y.VIVEROS, Trad.). Bogotá, Colombia: Editorial. KimpresUniversidad Libre.

GONZALEZ, J. L. \& GORRIZ, E. (2003). La Corte Penal Internacional (un estudio interdisciplinar). Valencia, España: Editorial Tirant lo Blanch.

PENAGOS, S. C. \& SANCHEZ, J. C. (2007). El non bis in ídem y la Cosa Juzgada en el Estatuto de Roma de la Corte Penal Internacional. Bogotá, Colombia: Grupo Editorial Ibáñez.

SCHABAS, W. (1999). Principios Generales del Derecho Penal en el Estatuto de la Corte Penal Internacional. El Estatuto de Roma de la Corte Penal Internacional. Bogotá, Colombia: Editorial Universidad Externado de Colombia. 
SOLORZANO, C. R. (2002). Unidad y Pluralidad de Acción, el Concurso de Conductas Punibles (1ra, Ed.). Bogotá, Colombia: Ediciones Nueva Jurídica

VELASQUEZ, F. (2002). Manual de Derecho Penal Parte General. Bogotá, Colombia: Editorial Temis.

\section{Jurisprudencia y normatividad}

CODIGO PENAL Y DE PROCEDIMIENTO PENAL. Anotado. Ley 599 de 2000 y ley 906 de 2004 de Colombia. Ed. Leyer. Bogotá D.C. 2008

COMPILACIÓN DE DERECHO INTERNACIONAL DE LOS DERECHOS HUMANOS. Oficina en Colombia del Alto comisionado de las Naciones Unidas para los Derechos Humanos. Bogotá D.C. 2004.

CORTE CONSTITUCIONAL, Sentencia C-004 de 20 de enero de 2003 M.P. Eduardo Montealegre Lynett.

CORTE CONSTITUCIONAL COLOMBIANA. (1994). Sentencia C-221.

CORTE CONSTITUCIONAL COLOMBIANA. (2002). M.P. CEPEDA, M. J. Sentencia C-578.

CORTE CONSTITUCIONAL COLOMBIANA. (2003). M.P. MONTEALEGRE, E. Sentencia C-004.

CORTE CONSTITUCIONAL COLOMBIANA. (2007). M.P. CEPEDA, M. J. Sentencia C-291.

CORTE CONSTITUCIONAL COLOMBIANA. (2009). M.P. MENDOZA, G. E. Sentencia C-801.

CORTE SUPREMA DE JUSTICIA, Sala de Casación Penal. Sentencia de 25 de julio de 2007. Proceso 27383. MP. Yesid Ramírez Bastidas.

ESTATUTO DE ROMA DE 1998 (E.R.), para el Establecimiento de la Corte Penal Internacional. Entrada en vigor, el $1^{\circ}$ de julio de 2002. Para Colombia, la entrada en vigencia se produjo el $1^{\circ}$ de noviembre de 2002 , en virtud de la ley 742 de 2002.

LEY 1268 de 31 de diciembre de 2008. Por medio de la cual se aprueban las "reglas de procedimiento y prueba" y los "elementos de los crímenes de la Corte Penal Internacional", aprobados por la Asamblea de los Estados Parte de Corte Penal Internacional, en Nueva York, del 3 al 10 de septiembre de 2002 Diario Oficial No. 47.219 de 31 de diciembre de 2008.

\section{Páginas web}

DOCUMENTO ICC-02-05-01-07 De 27 de abril de 2007. Orden de detención librada por la Sala de Cuestiones Preliminares. Situación Darfur Sudán- En el caso AHMAD MUHAMAD HARUN. Disponible En http://www.icc-cpi.int/ iccdocs/doc/doc345893.PDF (Consultada el día 5.03.2009).

DOCUMENTO ICC-02-05-01-09 de 04 de marzo de 2009. Caso de OMAR HASSAN AHMAD AL BASHIR. Orden de detención librada por la Sala de Cuestiones Preliminares. Situación Darfur Sudán- Disponible en http:// www.icc-cpi.int/iccdocs/doc/doc644487.pdf (Consultada el día 05.05.2009).

DOCUMENTO ICC-01/04-01/06-2-tEN 0304-2006 2/5 UM PT - Warrant of Arrest. The Prosecutor Vs Thomas Lubanga Dyilo. Disponible en: www.icc-cpi.int/iccdocs/doc/ doc191959.PDF. Consultada el 17.10.10

DOCUMENTO: International Tribunal for the Prosecution of Persons Responsible for Serious Violations of International Humanitarian Law Committed in the Territory of the Former Yugoslavia since 1991Case No.: IT-94-1-A and IT-94-1-Abis Date: 26 January 2000. Disponible en: www.icty.org/x/cases/tadic/ acjug/en/tad-asj000126e.pdf

EL TIEMPO. (2009, marzo). Hay 183 procesos por abuso sexual a más de 500 Mujeres en medio del Conflicto, Revela la Fiscalía. Consultado el 2 de marzo de 2009, de sitio Web de El Tiempo: www.eltiempo.com.co.

Dusco Tadić-. (IT-94-1) "Prijedor". -CaseInformation-Sheet.www.icty.org/x/cases/tadic/ cis/en/cis_tadic_en.pdf. 